\title{
Differential Regulation of Synaptic Vesicle Protein Genes by Target and Synaptic Activity
}

\author{
Jeffery A. Plunkett, ${ }^{1}$ Stephen A. Baccus, ${ }^{2}$ and John L. Bixby ${ }^{1,2}$ \\ ${ }^{1}$ Department of Molecular and Cellular Pharmacology and 2Neuroscience Program, University of Miami School of \\ Medicine, Miami, Florida 33136
}

\begin{abstract}
Differentiation of presynaptic nerve terminals involves changes in gene expression; these may be regulated by synaptic transmission and/or by contact with the target muscle. To gain insight into the control of presynaptic differentiation, we examined the regulation by target and synaptic activity of synaptic vesicle protein (SVP) genes in the chick ciliary ganglion (CG). In the CG, two SVP genes, synaptotagmin I (syt I) and synaptophysin II (syp II), are coordinately upregulated at the time of target contact. To test the hypothesis that this upregulation is induced by target contact, we examined mRNA levels of syt I and syp II in CGs from embryos in which one eye had been removed before axon outgrowth. As expected, target removal prevented the normal upregulation of syt I mRNA in the deprived ganglion. In contrast, and unexpectedly, syp II mRNA
\end{abstract}

upregulation was not affected. The target dependence of syt I upregulation was not attributable to nerve-muscle transmission, because blockade of this transmission had no effect on SVP mRNA levels. Surprisingly, blockade of synapses onto CG neurons from the brain also did not affect syt I mRNA levels but increased levels of syp II mRNA. We conclude that contact with target induces upregulation of syt I mRNA, which is the case for spinal motor neurons. However, the normal upregulation of syp II mRNA is not controlled by the same signal(s). Instead, our results suggest that these two SVP genes are differentially regulated, both by target contact and by blockade of synaptic transmission.

Key words: synaptotagmin; synaptophysin; ciliary ganglion; synapse formation; atropine; hemicholinium-3
Synaptogenesis at the neuromuscular junction requires coordinate differentiation of both the presynaptic and postsynaptic elements. For the presynaptic nerve terminal, this differentiation involves the reorganization of specific synaptic proteins (e.g., Bixby and Reichardt, 1985; Dai and Peng, 1996) as well as changes in the levels of expression of synapse-specific genes (e.g., Lou and Bixby, 1993, 1995). Relatively little is known concerning the mechanisms through which these changes in gene expression are regulated.

The most well-studied group of nerve terminal-specific proteins consists of the synaptic vesicle proteins (SVPs). The SVPs generally comprise families of related isoforms with different developmental and tissue distributions (Bajjalieh et al., 1994); prominent examples include the synapsins, the synaptophysins, and the synaptotagmins (Bahler et al., 1990; Bixby, 1992; Jahn and Sudhof, 1994; Li et al., 1995). In previous studies, we have used the expression of SVP genes as molecular markers of presynaptic differentiation (Lou and Bixby, 1993, 1995; Campagna et al., 1997). In the chick ciliary ganglion (CG), mRNAs encoding two SVPs, synaptophysin II (syp II) and synaptotagmin I (syt I), are

Received Oct. 27, 1997; revised April 24, 1998; accepted May 12, 1998.

This work was supported by the National Science Foundation Grant IBN 9603928 and by the National Institutes of Health Grant NS 34412 to J.L.B. J.A.P. was supported by a Cardiovascular Pharmacology Training Grant from the National Institute of Heart, Lung, and Blood (HL 01788). S.A.B. was supported by a Howard Hughes Medical Institute Predoctoral Fellowship. We thank Jason Campagna and the Jacob lab for invaluable advice on the techniques of optic vesicle extirpation, Ken Muller for generously allowing us to use physiology apparatus, and Chuck Luetje for his helpful comments on this manuscript.

Correspondence should be addressed to Dr. John L. Bixby, Department of Molecular and Cellular Pharmacology and Neuroscience Program, University of Miami School of Medicine, 1600 Northwest 10th Avenue, Miami, FL 33136.

Dr. Plunkett's present address: Miami Project to Cure Paralysis, University of Miami School of Medicine, 1600 Northwest 10th Avenue, Miami, FL 33136.

Copyright (C) 1998 Society for Neuroscience $0270-6474 / 98 / 155832-07 \$ 05.00 / 0$ upregulated at the time that these neurons contact their target muscle (Lou and Bixby, 1993, 1995). These results suggest that contact with muscle induces the upregulation of SVP genes in the innervating motor neurons. This hypothesis has been partially tested in the case of spinal motor neurons. It has been found that the increase in syt I mRNA within the lateral motor column, which normally occurs at the time of muscle contact, is abrogated by removal of this target before innervation (Campagna et al., 1997).

Our study with spinal motor neurons implies that at least one SVP gene depends on target contact for upregulation in this neuronal population. However, several important questions remain unanswered. Is syt I upregulated by target contact in other motor neuron populations, and are other SVP genes upregulated by target contact? Is synaptic activity between neuron and muscle required for upregulation of SVP genes? Are SVP genes regulated by synaptic signals impinging on the motor neuron itself?

We have addressed these issues using the chick CG in vivo as our motor neuronal population. We examined levels of mRNA for two SVPs, syt I and syp II, in CG neurons in vivo after various perturbations of normal development. These perturbations included (1) peripheral target (eye) removal before innervation and (2) application of drugs blocking either postganglionic (CGtarget) activity or both postganglionic and preganglionic (brain$\mathrm{CG}$ ) activity before and during the early innervation period. Our results suggest that levels of mRNA encoding syt I and syp II are not coordinately upregulated by contact between neurons and muscle targets as expected; instead, distinct mechanisms operate to regulate these two SVP genes in developing CG neurons.

\section{MATERIALS AND METHODS}

Materials. Fertilized White Leghorn eggs were purchased from SPAFAS (Norwich, CT) and incubated in a humidified forced-draft incubator 
until the desired stage of development. All RNase-free reagents and atropine sulfate were purchased from Sigma (St. Louis, MO). Hemicholinium-3 was obtained from Aldrich (Milwaukee, WI).

Surgical manipulations. Chick embryos from embryonic day 2 (E2) (stage 13) to E10 (stage 36) were used in this study. All staging was done by the staging methods of Hamburger and Hamilton (1951). Unilateral eye removals were performed at E2 (stages 10-13), following previously described methods (Dourado et al., 1994). Briefly, the egg was windowed, and the extraembryonic membranes were opened just enough to expose the head of the developing embryo. Finely sharpened forceps were then used to remove the developing optic vesicle unilaterally. The window was resealed with Scotch Magic tape $(3 \mathrm{M})$, and the embryos were placed back into the incubator until E8. Embryos were analyzed only if no trace of eye tissue was observed on the operated side at the time of harvest.

Drug treatments. All drugs were dissolved in sterile isotonic saline and were delivered in doses of $100 \mu \mathrm{l}$ per dose either once or twice daily from E6 to E8.5. Eggs were windowed at E5; after the shell and extraembryonic membranes were perforated, the egg was resealed with Scotch Magic tape (3M). A formula from Pilar et al. (1987) was used to determine the correct dosage of atropine. Briefly, effective concentrations of drug were determined assuming uniform diff usion with an estimated egg volume of $40 \mathrm{ml}$. A dose of $0.15 \mathrm{mg} / \mathrm{dose} / \mathrm{d}$ (Meriney et al., 1987) (estimated final concentration, $5 \mu \mathrm{M}$ ) was applied to the chorioallantoic membrane of the embryo. Hemicholinium-3 was applied to windowed embryos as described at a dose of $1 \mathrm{mg} /$ dose (twice daily; estimated final concentration, $40 \mu \mathrm{M}$ ) (Maderdrut et al., 1988). Controls for both drug experiments involved windowing the egg and removing the shell membrane at E5, followed by application of $100 \mu \mathrm{l}$ of saline (once or twice daily) from E6 to E8.5.

Assays of drug efficacy. The efficacy of atropine treatment was measured at E10, after application of atropine from E6 to E10 (stages 29-36), using an iris contraction assay originally developed by Pilar et al. (1987). Briefly, eyes with intact, attached CGs were isolated from both drugtreated and saline controls at $6 \mathrm{hr}$ after the final injection. Iris contraction was measured (with a red-filtered light) using a dissecting microscope with an eyepiece micrometer either after electrical stimulation $(20 \mathrm{sec}$ at $50 \mathrm{~Hz}$ ) through a suction electrode attached to the ganglion or after bathing the iris in $100 \mathrm{~mm} \mathrm{KCl}$ for direct contraction. The preparation for stimulation has been described previously (Pilar et al., 1987).

The efficacy of hemicholinium-3 (HC-3) treatment was measured by use of a leg kick assay for neuromuscular synapses and by measurements of transmission through the CG. Leg kick measurements were made at $\sim 9 \mathrm{hr}$ after the first E8 injection and before the second E8 dose. A window was opened over the embryo, and the frequency of discrete spontaneous hindlimb kicks over a $5 \mathrm{~min}$ period was measured. Synaptic transmission through the CG was measured using methods similar to those of Landmesser and Pilar (1974a), with ganglia from E8 to E9 embryos.

RNase protection assay. RNA isolation and RNase protection assays were performed as described previously (Lou and Bixby, 1993, 1995). Modifications to the original RNase protection assay procedures include the following. A new syt I probe was generated from a $B g l \mathrm{II} / \mathrm{Ns} \mathrm{I}$ digest of clone p65.1 (Lou and Bixby, 1993) [nucleotides (nt) 1279-1590 of the published sequence]. This piece was cloned into the pSP73 vector (Promega, Madison, WI) in the antisense orientation relative to the SP6 promoter. This resulted in a probe size of $355 \mathrm{nt}$ and a protected fragment of $312 \mathrm{nt}$. This method of probe preparation allowed for individual preparations of total RNA to be analyzed simultaneously for levels of syp IIa, syp IIb, syt I, $\beta$-actin, and neurofilament-M mRNAs (see Fig. 1). Signals were quantified from autoradiographic film using SigmaScan Pro (Jandel Scientific, Corte Madera, CA). Multiple autoradiographic exposures were used to ensure that signals were in the linear range.

Normalization of $m R N A$ signals. In a previous study, we outlined our rationale for the use of neurofilament-M (nfM) mRNA as a normalization standard rather than absolute mRNA amount, neuron number, ganglion number, or $\beta$-actin mRNA (Lou and Bixby, 1995). As a test of this justification, we have compared the relative levels of syp II (IIa + IIb) mRNA at E7 and E9 using three different methods of normalization. Normalizing syp II mRNA to the number of ganglia (equivalent to neuron number at this time) yielded a sixfold apparent upregulation between E7 and E9, whereas normalizing to $\beta$-actin (2.5-fold) or nfM (threefold) gave results approximately comparable with each other (data not shown). We conclude that either $\beta$-actin or nfM mRNA is a suitable normalization standard, with nfM providing the most specific test (Lou and Bixby, 1995), whereas normalization to ganglion or neuron number overestimates the magnitude of specific SVP mRNA upregulation. In this study, therefore, the relative amounts of syt I and syp II mRNAs were normalized to $\mathrm{nfM}$ and/or to $\beta$-actin mRNA.

\section{RESULTS SVP mRNA expression in the CG after unilateral
eye removal}

To test the hypothesis that increased levels of SVP mRNAs are induced by contact of CG neurons with their targets, we examined the levels of syt I and syp II mRNAs after unilateral eye removal. The developing optic vesicle was removed before CG formation (E2; stage 13-19), and the animals were allowed to develop until E8 (stage 34), $\sim 1$ d after contact of CG neurons with their targets. Examination at this stage eliminates the interpretational difficulties caused by cell death; morphological changes associated with cell death are first observed in target-deprived CG neurons at E9 (stage 35) (Landmesser and Pilar, 1974a, 1976). Before the period of cell death, synaptic transmission and ultrastructure of the CG neurons are normal (Landmesser and Pilar, 1974b).

Our laboratory has shown previously that mRNA expression levels for syt I and for syp II are upregulated approximately threefold (2.5-fold for syt I) within 2 d (E7-E9; stages 31-35) of contact with targets in the eye and that these are the only significant increases in mRNA expression during embryonic development (Lou and Bixby, 1993, 1995). In those studies, we did not measure mRNA levels at E8. To test whether SVP mRNAs are upregulated as early as E8, we measured mRNAs for syt I and syp II (IIa + IIb), normalized to that for nfM, in CG neurons at E7 and E8. In these experiments we found that syp II mRNA was 2.4-fold higher and that syt I mRNA was twofold higher at E8 compared with E7 (data not shown). Therefore, $\sim 80 \%$ of the mRNA upregulation seen during development has occurred by $1 \mathrm{~d}$ after target contact (E8; stage 34). We conclude that examination of eye-deprived ganglia at E8 will allow us to test for the target dependence of gene upregulation.

Because it was difficult to obtain sufficient material from eyedeprived ganglia to use nfM as the normalization standard, we used $\beta$-actin in most experiments. As expected, there was a 1.9-fold decrease in the expression of syt I mRNA relative to that in the contralateral controls, almost quantitatively accounting for the normal developmental increase (Fig. 1B). This result implies that syt I gene upregulation in CG neurons completely depends on the presence of the synaptic target, as is the case for spinal motor neurons (Campagna et al., 1997). Surprisingly, however, there were no significant differences in the levels of syp IIa or syp IIb between the controls and eye-deprived ganglia (Fig. 1). Indeed, the data show a slight trend toward higher levels on the deprived side for syp IIa. To ensure that our results were not biased by the use of $\beta$-actin normalization, we performed two experiments using nfM mRNA as the standard. In these experiments, syt I mRNA levels in the eye-deprived CG were decreased by the same amount that was seen with the actin experiments (1.8-fold), whereas levels of syp IIa and syp IIb mRNA were not significantly changed, when compared with the contralateral controls. Although these data must be considered less reliable than are the data normalized to actin, they confirm that the syt I mRNA upregulation in developing CG is target-dependent and that syp II mRNAs are not upregulated by the same mechanism. 
A. B.
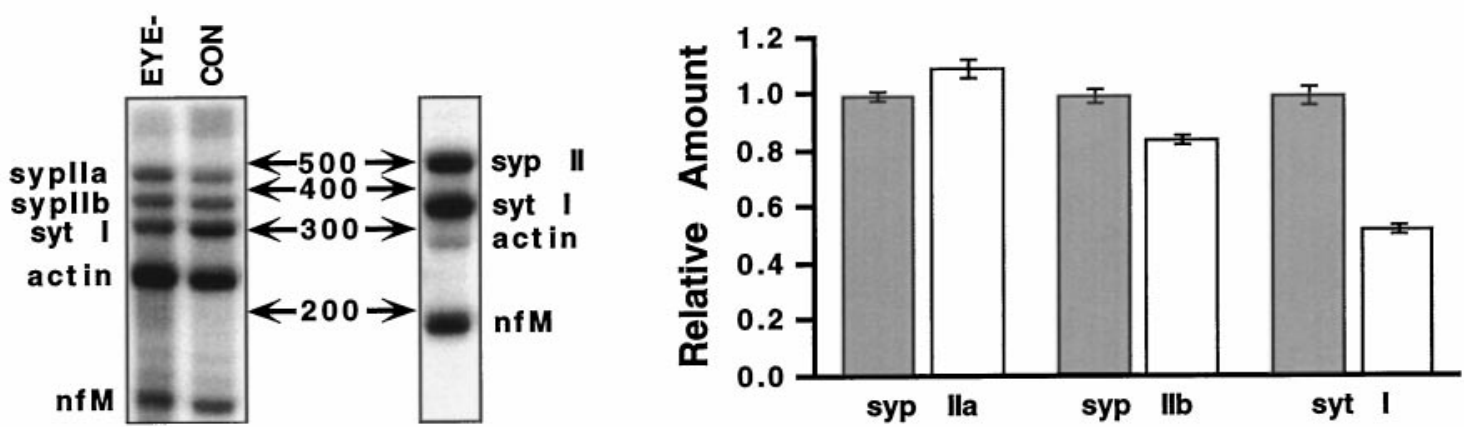

Figure 1. Expression levels of SVP mRNAs in control and target-deprived CGs at E8. A, Autoradiogram of an RNase protection assay. On the right, the probes used in the assay are shown, and the corresponding signals from the protected fragments are shown on the left. EYE-, CGs ipsilateral to the removed eye; $C O N$, CGs contralateral to the removed eye. The positions of molecular weight markers (in nt) are shown with arrows. Note that the syt $I$ signal is reduced in the $E Y E$ - lane, even though the actin and $n f M$ signals are greater. $B$, Relative levels of SVP mRNAs in contralateral control (shaded bars) and target-deprived (open bars) CGs, quantified from four experiments with 12-18 ganglia per condition per experiment (mean \pm SEM). The levels of syt I, syp IIa, and syp IIb mRNA are shown normalized to that of actin. Note that syp II mRNA levels are not affected by the absence of target but that syt $I$ mRNA levels are reduced 1.9 -fold $(p<0.001)$. Qualitatively similar results were obtained in two experiments using $n f M$ mRNA as a standard (see text).

\section{SVP mRNA expression after blockade of peripheral synaptic activity}

Given the target dependence of syt I mRNA upregulation, it is natural to wonder whether "target contact" in this context requires synaptic activity. Maturation of the synaptic apparatus at neuromuscular junctions seems to depend on neuromuscular activity (Lohof et al., 1993; Poo, 1994; Xie et al., 1997). Therefore, it is reasonable to suppose that the upregulation of SVP genes, a presumed requirement for this maturation, could be linked to neuromuscular transmission.

The ciliary ganglion contains two types of cholinergic neurons: ciliary neurons and choroid neurons. Synaptic transmission from choroid neurons to smooth muscle in the choroid layer is muscarinic. Although transmission from ciliary neurons to mature iris muscle is nicotinic, only muscarinic ACh receptors are functional on iris muscle before E11 (stage 37) (Pilar et al., 1987). Therefore, the muscarinic agonist atropine would be expected to block transmission from both neuronal types between E6 and E10 (Fig. 2). To investigate the role that neuromuscular activity plays in syt I mRNA upregulation in the CG, we delivered an atropine solution daily to the chorioallantoic membrane of embryos beginning at E6 (stage 29) and harvested the CGs at E9 (stage 35), 15 $\mathrm{hr}$ after the final injection. These ages were chosen because they span the time from first peripheral contact of CG axons (Meriney and Pilar, 1987) to the time of SVP gene upregulation. To test the efficacy of the atropine blockade, we examined CG-evoked contraction of the iris in control and atropine-treated embryos $6 \mathrm{hr}$ after a final in ovo application. In these experiments, atropinetreated animals showed a 79\% reduction in iris contraction when compared with saline controls (Table 1). This is likely to be an underestimate of the in ovo effect, because the eyes were bathed in normal saline during the experiments and atropine could have begun to wash out. Our results confirm the results of Pilar et al. (1987), showing that transmission to the iris at early stages is muscarinic, and demonstrate that we established an atropine dose that is able to block substantially synaptic transmission to the iris from the CG.

Blockade of transmission from CG neurons to their muscle targets had no effect on the levels of either syt I or syp II mRNAs
(Fig. 3). These data suggest that the target dependence of syt I mRNA upregulation is not attributable to functional synaptic transmission. Rather, the target-derived signal seems effective in the absence of synaptic activity.

\section{Syt I and syp II mRNA expression in the presence of ganglionic and peripheral activity-blocking drugs}

The normal developmental increase in syp II mRNA does not depend on contact with muscle targets or synaptic activity. One possibility is that the increase in syp II mRNA is induced by synaptic activity from preganglionic neurons in the accessory oculomotor nucleus. There are numerous examples of genes in postsynaptic neurons being upregulated by activity from innervating neurons (e.g., Black et al., 1985). To elucidate the role that preganglionic activity may play in the upregulation of syp II and syt I mRNAs, we applied HC-3 to embryos in ovo. HC-3 prevents the reuptake of choline from the synaptic cleft into the presynaptic terminal and thereby prevents cholinergic nerve terminals from synthesizing and secreting acetylcholine. Because CG neurons are both cholinoceptive and cholinergic, HC-3 should effectively block both pre- and postganglionic transmission (Fig. 2).

We applied HC-3 in ovo, beginning at E6 (stage 29; the time at which CG neurons begin to receive synapses) (Landmesser and Pilar, 1972) and continuing to E8.5 (stage 34), and examined the CGs at E9 (stage 35), $0.5 \mathrm{~d}$ after the final HC-3 injection. Because HC-3 affects cholinergic transmission in general, we could test its efficacy by examining peripheral neuromuscular activity, as measured by the frequency of spontaneous hindlimb kicks. When we examined embryos $9 \mathrm{hr}$ after injection (at E8), we found that HC-3 reduced hindlimb kicks from a control value of $9.9 \pm 1.5$ kicks $/$ min (mean $\pm \mathrm{SEM} ; n=4$ embryos) to $2.6 \pm 0.5 \mathrm{kicks} / \mathrm{min}$ $(n=5)$, a reduction of $76 \%$. It was also clear that overall movement of the embryos was considerably reduced by the drug treatment, although this was not quantified. Thus, our HC-3 dose largely inhibited neuromuscular activity during this period.

We examined SVP mRNA levels from HC-3-treated CGs using $\mathrm{nfM}$ as the normalization standard. As was the case for blockade of postganglionic activity alone, there was no effect of the HC-3 treatment on levels of syt I mRNA (Fig. 4). Therefore, 


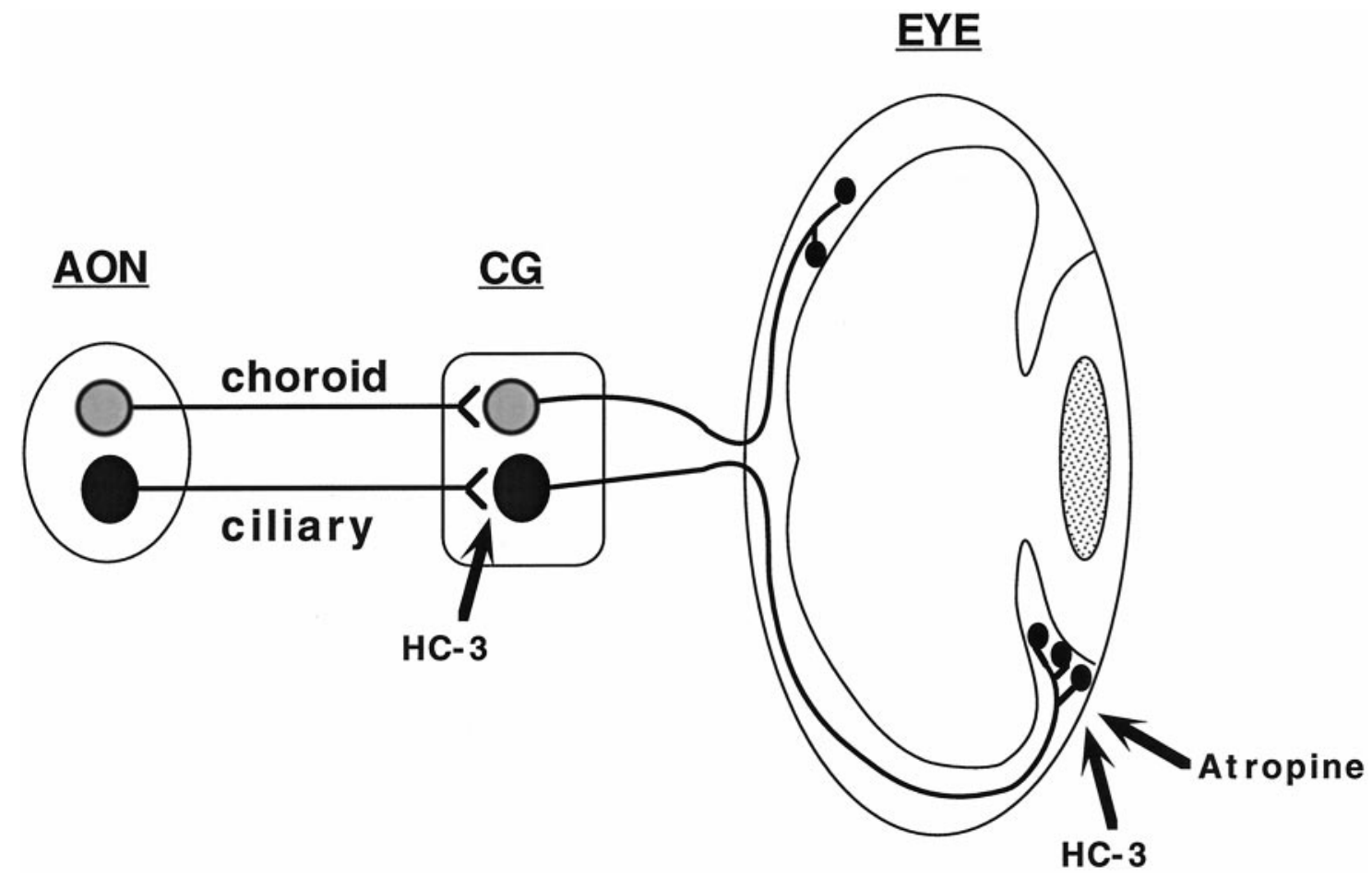

Figure 2. Schematic of the neural circuit involving the $C G$ and the sites of drug action (arrows) for atropine and $H C$-3. Brainstem neurons from the accessory oculomotor nucleus $(A O N)$ make nicotinic cholinergic synapses onto both types of neurons (ciliary and choroid) in the $C G$. In turn, $C G$ neurons send axons in the postganglionic nerve to make cholinergic synapses onto targets in the eye, which include the iris and ciliary body for the ciliary neurons and smooth muscle in the choroid for the choroid neurons. Before E10, when our experiments were performed, both ciliary and choroid postganglionic synapses are blocked by atropine. $H C$-3, by depleting neurons of acetylcholine, blocks both pre- and postganglionic synapses.

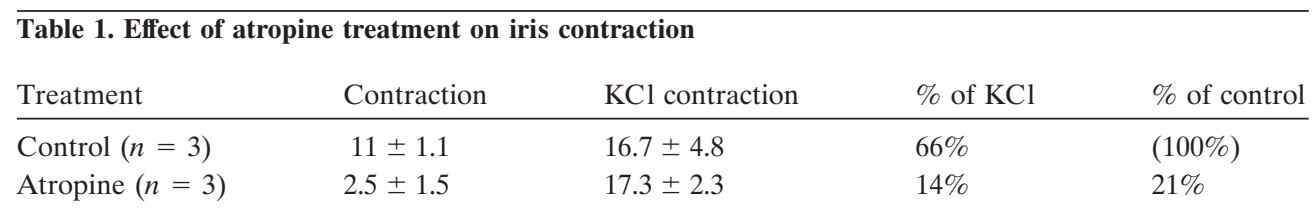

Embryos were treated with atropine according to the standard protocol, and eyes with intact CGs were removed 6 hr after the final atropine injection. Contraction refers to that elicited by electrical stimulation of the $\mathrm{CG}$, and $\mathrm{KCl}$ contraction refers to that elicited directly by application of $\mathrm{KCl}$ to the bathing solution. Contraction data are expressed as a percent reduction of the total pupillary diameter (mean $\pm \mathrm{SEM})$.

the target-dependent elevation of syt I mRNA does not require pre- or postganglionic synaptic activity. Surprisingly, however, HC-3 treatment not only did not reduce syp II levels but actually resulted in increases in syp II mRNAs (Fig. 4). Syp IIa mRNA levels were elevated 1.8 -fold in drug-treated CGs $(p<0.05)$, whereas syp IIb levels increased $\sim 1$.7-fold ( $p<0.01$ ). Therefore, syp II mRNAs are upregulated by cholinergic blockade, whereas levels of syt I mRNA are unchanged.

Because the upregulation of syp II mRNA was unexpected, we examined directly whether synaptic transmission in the CG was blocked by our drug-treatment protocol. This was accomplished by in vitro stimulation of the preganglionic nerve while recording from the postganglionic nerve. In control ganglia, preganglionic stimulation produced action potential activity that could be recorded postsynaptically ( $n=2 / 2$ ganglia), and this was abolished by incubation in a low $\mathrm{Ca}^{2+}$ and high $\mathrm{Mg}^{2+}$ solution (Fig. 5). In ganglia treated with $\mathrm{HC}-3$ for $3 \mathrm{~d}$ and examined at $\mathrm{E} 9$, this synaptically evoked activity was absent ( $n=3 / 3$ ganglia; Fig. 5). This result was not attributable to nerve damage, because distal stimulation of the postganglionic nerve produced a large com- pound action potential (Fig. 5). Therefore, our HC-3 treatment effectively blocked synaptic activity in the CG.

\section{DISCUSSION}

In the chick CG, at least two different SVPs, syt I and syp II, are upregulated at the time of contact with peripheral targets (Lou and Bixby, 1993, 1995), and results with spinal motor neurons suggested that these upregulations were likely target-dependent, at least in the case of syt I (Campagna et al., 1997). Our present results demonstrate that, as expected, upregulation of syt I mRNA in the CG depends on the presence of the synaptic target. In addition, we have extended these results to show that neither pre- nor postganglionic synaptic activity is required for this target-dependent gene upregulation. Contrary to our expectations, however, syp II mRNA is not regulated in the same way. Not only is the developmental increase in syp II mRNA independent of target contact, but these mRNAs, unlike that for syt I, are upregulated after blockade of pre- and postganglionic transmission with HC-3. The surprising conclusion is that syt I and syp II 

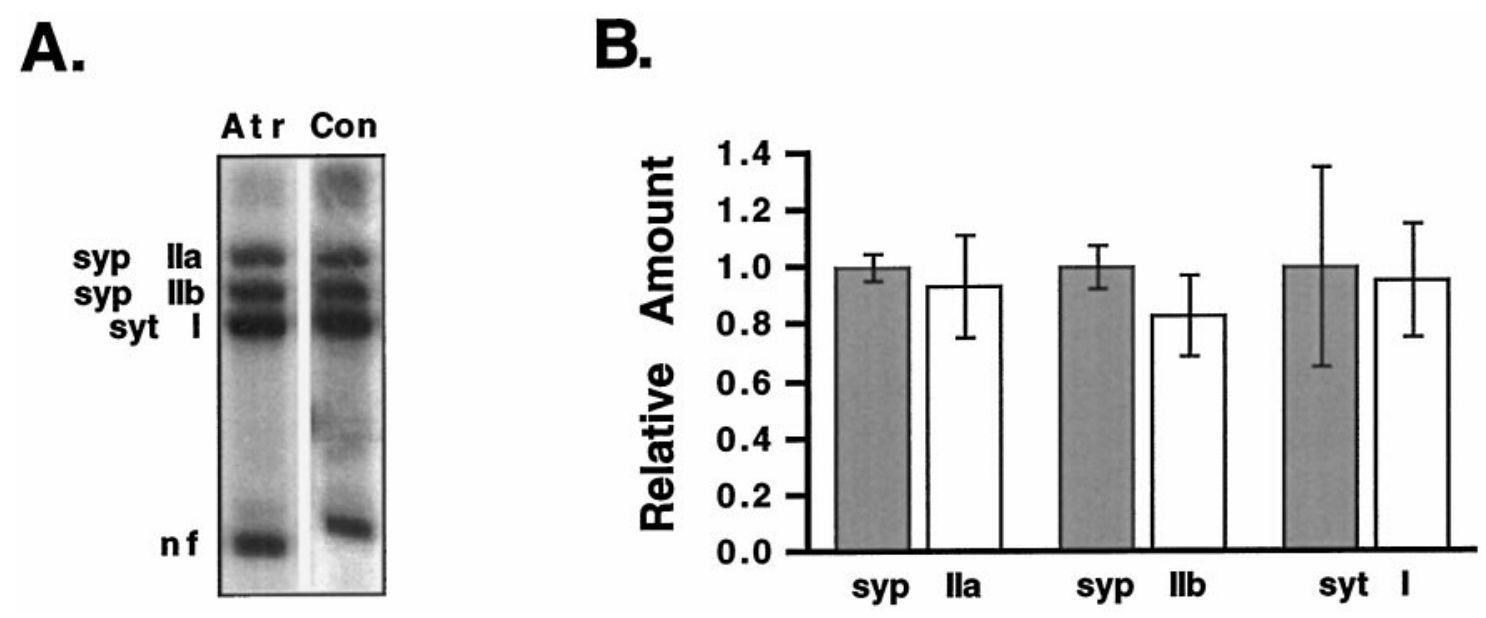

Figure 3. Expression levels of SVP mRNAs in control and atropine-treated CGs at E9. A, Autoradiogram of an RNase protection assay. Signals are approximately equal in the control (Con) and atropine-treated (Atr) lanes. $n f$, Neurofilament-M. B, Relative levels of SVP mRNAs in control (shaded bars) and atropine-treated (open bars) CGs, quantified from two experiments with 20-30 ganglia per condition per experiment (mean \pm range). The levels of syt $I$, syp IIa, and syp IIb mRNAs are shown normalized to that of $n f$ mRNA. Note that RNA levels for all three SVPs are unaffected by the block of peripheral synaptic activity.

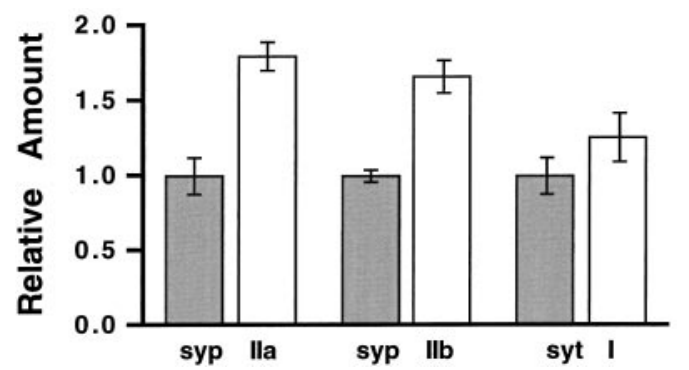

Figure 4. Expression levels of SVP mRNAs in control and HC-3treated CGs at E9. Relative levels of SVP mRNAs are shown for control (shaded bars) and HC-3-treated (open bars) CGs, normalized to nfM, quantified from four experiments with 20-30 ganglia per condition per experiment (mean \pm SEM). Both syp IIa $(p<0.05)$ and syp IIb $(p<$ $0.01)$ are significantly elevated by the HC-3 treatment. There is no significant change in syt $I$ mRNA. Similar results were seen in two experiments in which the data were normalized to $\beta$-actin, although syp II mRNA increases were not as great in the case with $\beta$-actin (data not shown).

mRNAs are not coordinately regulated, either by synaptic activity or by contact with peripheral targets.

Several key strengths of this work deserve mention. First, because we found that upregulation of CG SVP genes is substantial by E8, we were able to work within a developmental time frame that avoided complications caused by neuronal cell death. Second, our normalization to nfM (and to $\beta$-actin) ensured that our measured changes in SVP mRNAs were selective and not attributable to generalized neuronal growth or neuronal differentiation. Third, our peripheral blockade was achieved via the use of atropine, which blocks both ciliary and choroid transmission at these early time points, in contrast to nicotinic blocking agents such as $\alpha$-bungarotoxin. Finally, we applied activity-blocking drugs during a short, relevant developmental window, minimizing the risk of irrelevant side effects of the drugs.

Combined with the earlier study of Campagna et al. (1997), our data demonstrate the existence of target-dependent developmental increases in syt I mRNA in two separate populations of motor neurons. This confirms (for one SVP) our original hypothesis (Lou and Bixby, 1993) that contact of motor neurons with pe- ripheral targets would result in upregulation of synapse-specific genes. Clearly, it would be of interest to know whether this kind of target-mediated upregulation occurs at neuron-neuron synapses as well.

A number of studies have examined the developmental regulation of neuronal genes in the CG (e.g., Dourado et al., 1994; Bruses et al., 1995; Levey et al., 1995; Thomas et al., 1995). In particular, our data add to existing evidence from the Jacob and Dryer laboratories that contact with peripheral targets is required for normal expression of neuron-specific genes in the CG. Levey et al. (1995) have shown that loss of peripheral targets reduces levels of mRNA encoding $\alpha 3$ and $\beta 4 \mathrm{ACh}$ receptor (AChR) subunits in the CG, without changing levels of $\alpha 5$ mRNA. Similarly, Dourado et al. (1994) showed that loss of target prevented the normal expression of the $\mathrm{Ca}^{2+}$-dependent $\mathrm{K}^{+}$current in $\mathrm{CG}$ neurons and significantly reduced a component of slow inactivation of the A-type $\mathrm{K}^{+}$current. Therefore, contact with muscle targets is required for the normal upregulation of at least three distinct types of neuronal genes, two of which are clearly related to synapse formation. Interestingly, in both of the above cases, as in the present study, target-dependent upregulation was found for some, but not all, of the genes encoding proteins with similar functions (SVPs, AChR subunits, and $\mathrm{K}^{+}$channels).

Syt I mRNA, but not syp II mRNAs, requires the presence of target for its developmental upregulation. In addition, syp II mRNAs, but not syt I mRNA, are upregulated after an HC-3mediated derangement of acetylcholine metabolism. These results indicate that the control mechanisms regulating mRNA expression are different for different SVP genes. Our initial assumption, therefore, that genes encoding membrane proteins of synaptic vesicles would be coregulated is evidently incorrect. It seems reasonable to suppose that this differential regulation of SVP genes could be related to different nonsynaptic functions of the corresponding proteins. For example, other proteins involved in vesicle exocytosis seem to regulate axon growth as well (OsenSand et al., 1993; Igarashi et al., 1996).

Our experiments lead to two important questions concerning the regulation of syp II mRNA. First, if neither contact with peripheral targets nor synaptic activity are required for develop- 


\section{$\underline{\text { Control }}$}

A

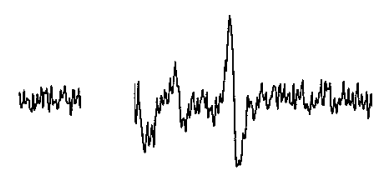

C

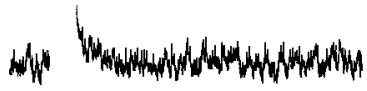

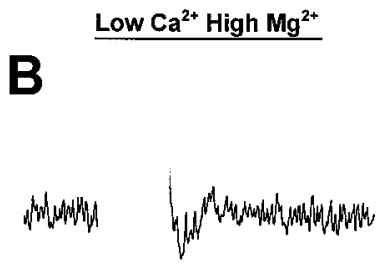

D

My

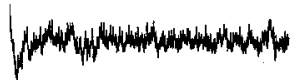

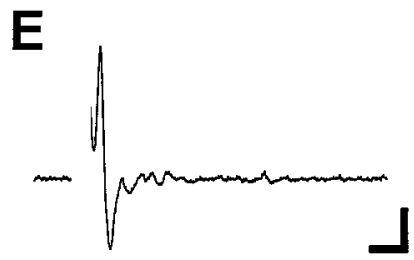

Figure 5. Blockade of transmission through the CG by treatment with HC-3. Suction electrodes were used for stimulation and recording from isolated $\mathrm{CG}$ examined in vitro. $A, B$, In untreated embryos, preganglionic stimulation (blank area in trace) produced action potential activity $(A)$. This evoked activity was abolished after incubation in saline containing $0.1 \mathrm{~mm} \mathrm{Ca}{ }^{2+}$ and $6 \mathrm{mM} \mathrm{Mg}^{2+}(B)$. $C-E$, Similar preganglionic stimulation of CG from HC-3-treated embryos evoked no activity, either in normal saline $(C)$ or in low $\mathrm{Ca}^{2+}$ and high $\mathrm{Mg}^{2+}(D)$, despite the fact that spontaneous action potentials could sometimes be seen in these nerves (data not shown) and that distal stimulation of the postganglionic nerve produced a robust compound action potential $(E)$. Calibration: $A-D, 20 \mu \mathrm{V}, 5 \mathrm{msec} ; E, 120 \mu \mathrm{V}, 5 \mathrm{msec}$.

mental upregulation, how is this upregulation achieved? Second, how does synaptic blockade with HC-3 lead to upregulation of syp II mRNAs? Although we cannot answer the first question, some clues may be found in consideration of the second question. We can think of two broad categories of mechanism for the HC-3 effect on syp II mRNAs. First, synaptic drive from preganglionic inputs may be inhibitory to syp II mRNA expression (or the loss of such drive could signal mRNA upregulation). In either case, the effect of HC-3 would be to promote upregulation by blocking preganglionic synapses. Second, there could be some kind of negative coupling between synthesis and/or release of acetylcholine by CG neurons and the expression in these neurons of syp II mRNAs. In this case, the effect of $\mathrm{HC}-3$ would be to upregulate syp II mRNA via the inhibition of acetylcholine synthesis and/or release by the CG neurons themselves. We cannot distinguish among these and other possibilities at present.

Our experiments on regulation of SVP genes in motor neurons (Lou and Bixby, 1993, 1995; Campagna et al., 1997; this study) have been driven by the hypothesis that, during development, contact with synaptic targets should lead to the upregulation of SVP genes. Where does this hypothesis stand? In the case of syt I, our evidence suggests that the hypothesis is correct for two different populations of motor neurons. However, it is now clear that not all SVP genes are regulated by target contact at the level of mRNA expression. Given that mature synapses require relatively large amounts of SVPs, how might these levels be achieved during synapse formation and maturation for proteins like syp II? One recent study suggests that, at least in vitro, developmental upregulation of synaptophysin (a close relative of syp II) occurs mainly at the level of initiation of protein translation (Daly and Ziff, 1997). If this is true in vivo, it may be that target regulation of SVP expression is indeed a general phenomenon but that different mechanisms are used in different situations to achieve this general goal.

\section{REFERENCES}

Bahler M, Benfenati F, Valtorta F, Greengard P (1990) The synapsins and the regulation of synaptic function. Bioessays 12:259-263.

Bajjalieh SM, Frantz GD, Weimann JM, McConnell SK, Scheller RH
(1994) Differential expression of synaptic vesicle protein 2 (SVP2) isoforms. J Neurosci 14:5223-5235.

Bixby JL (1992) Identification of an alternatively spliced avian member of the synaptophysin gene family. Mol Brain Res 13:339-348.

Bixby JL, Reichardt LR (1985) The expression and localization of synaptic vesicle antigens at neuromuscular junctions developing in vitro. J Neurosci 5:3070-3080.

Black IB, Chikaraishi DM, Lewis EJ (1985) Trans-synaptic increase in RNA coding for tyrosine hydroxylase in a rat sympathetic ganglion. Brain Res 339:151-153.

Bruses JL, Oka S, Rutishauser U (1995) NCAM-associated polysialic acid on ciliary ganglion neurons is regulated by polysialyltransferase levels and interaction with muscle. J Neurosci 15:8310-8319.

Campagna JA, Prevette D, Oppenheim RW, Bixby JL (1997) Target regulates expression of synaptotagmin genes in spinal motor neurons in vivo. Mol Cell Neurosci 8:377-388.

Dai Z, Peng HB (1996) Dynamics of synaptic vesicles in cultured spinal cord neurons in relationship to synaptogenesis. Mol Cell Neurosci 7:443-452.

Daly C, Ziff EB (1997) Post-transcriptional regulation of synaptic vesicle protein expression and the developmental control of synaptic vesicle formation. J Neurosci 17:2365-2375.

Dourado MM, Brumwell C, Wisgirda ME, Jacob MH, Dryer SE (1994) Target tissues and innervation regulate the characteristics of $\mathrm{K}^{+}$currents in chick ciliary ganglion neurons developing in situ. J Neurosci 14:3156-3165.

Hamburger V, Hamilton HL (1951) A series of normal stages in the development of the chick embryo. J Morphol 88:49-92.

Igarashi M, Kozaki S, Terakawa S, Kawano S, Ide C, Komiya Y (1996) Growth cone collapse and inhibition of neurite growth by botulinum neurotoxin C1: a t-SNARE is involved in axonal growth. J Cell Biol 134:205-215.

Jahn R, Sudhof TC (1994) Synaptic vesicles and exocytosis. Annu Rev Neurosci 17:219-246.

Landmesser L, Pilar G (1972) The onset and development of transmission in the chick ciliary ganglion. J Physiol (Lond) 222:691-713.

Landmesser L, Pilar G (1974a) Synapse formation during embryogenesis on ganglion cells lacking a periphery. J Physiol (Lond) 241:715-736.

Landmesser L, Pilar G (1974b) Synaptic transmission and cell death during normal ganglionic development. J Physiol (Lond) 241:737-749.

Landmesser L, Pilar G (1976) Fate of ganglionic synapses and ganglion cell axons during normal and induced cell death. J Cell Biol 68:357-374.

Levey MS, Brumwell CL, Dryer SE, Jacob MH (1995) Innervation and target tissue interactions differentially regulate acetylcholine receptor 
subunit mRNA levels in developing neurons in situ. Neuron 14:153-162.

Li C, Ullrich B, Zhang JZ, Anderson RG, Brose N, Sudhof TC (1995) $\mathrm{Ca}(2+)$-dependent and -independent activities of neural and nonneural synaptotagmins. Nature 375:594-599.

Lohof AM, Ip NY, Poo MM (1993) Potentiation of developing neuromuscular synapses by the neurotrophins NT-3 and BDNF. Nature 363:350-353.

Lou XJ, Bixby JL (1993) Coordinate and non-coordinate regulation of synaptic vesicle protein genes during embryonic development. Dev Biol 159:327-337.

Lou XJ, Bixby JL (1995) Patterns of presynaptic gene expression define two stages of synaptic differentiation. Mol Cell Neurosci 6:252-262.

Maderdrut JL, Oppenheim RW, Prevette D (1988) Enhancement of naturally-occurring cell death in the sympathetic and parasympathetic ganglia of the chick embryo following blockade of ganglionic transmission. Brain Res 444:189-194.

Meriney SD, Pilar G (1987) Cholinergic innervation of the smooth muscle in the choroid coat of the chick eye and its development. J Neurosci $7: 3827-3839$
Meriney SD, Pilar G, Ogawa M, Nunez R (1987) Differential neuronal survival in the avian ciliary ganglion after chronic acetylcholine receptor blockade. J Neurosci 7:3840-3849.

Osen-Sand A, Catsicas M, Staple JK, Jones KA, Ayala G, Knowles J, Grenningloh G, Catsicas S (1993) Inhibition of axonal growth by SNAP-25 antisense oligonucleotides in vitro and in vivo. Nature 364:445-448

Pilar G, Nunez RR, McLennan IS, Meriney SD (1987) Muscarinic and nicotinic synaptic activation of the developing chicken iris. J Neurosci 7: 3813-3826.

Poo MM (1994) Activity-dependent modulation of developing neuromuscular synapses. Adv Second Messenger Phosphoprotein Res 29:521-527.

Thomas WS, Jacob MH, O’Dowd DK, Smith MA (1995) Agrin gene expression in ciliary ganglion neurons following preganglionic denervation and postganglionic axotomy. Dev Biol 168:662-669.

Xie K, Wang T, Olafsson P, Mizuno K, Lu B (1997) Activity-dependent expression of NT-3 in muscle cells in culture: implications in the development of neuromuscular junctions. J Neurosci 17:2947-2958. 